\title{
On the Locus of the Foci of a System of Similar Conics through three Points.
}

\author{
By R. E. Allardice.
}

Received January 1909. Read 12th February 1909.

1. The problem has been proposed by Steiner* of finding the envelope of a system of similar conics circumscribed about a given triangle, and of finding the loci of the centres and foci of the conics of the system. He states that the envelope is a curve of the fourth order having three double points, and gives some of its properties. The problem has been treated by P. H. Schoute in a paper entitled Application de la transformation par droites symétriques à un probleme de Steiner. $\dagger$ In this paper the author discusses the problem of the envelope in detail by a geometrical method, and gives the order of the locus of centres, of the locus of foci, and of the locus of vertices, and the class of the envelope of asymptotes, of the envelope of axes, and of the envelope of directrices. But in every case (except that of the locus of centres), he gives the degree or class twice as great as it should be. In a paper published in the Annals of Mathematics $\ddagger$ I have found explicit equations for the locus of centres and the envelope of asymptotes, showing that each asymptote envelopes a three-cusped hypocycloid; and in a paper in the Transactions of the American Mathematical Society, I

- Systematische Entwickelung der Abhängigkeit geometrischer Gestalten von einander (problem 39 of the supplement), Gesammelte Werke, vol. 1, p. 446 ; Vermischte Sätze und Aufgaben, ibid., vol. 2, p. 675.

$\dagger$ Bulletin des sciences mathématiques et astronomiques, ser. 2, vol. 7 (1883), pp. 314-324.

‡ Second series, vol. 3, No. 4 (1902), On some curves connected with a system of similar conics.

\$ Vol. 4, No. 1 (1903), On the envelope of the axes of a system of conics passing through three fixed points. 
have obtained an equation for the envelope of the axes, showing that each axis also envelopes a three-cusped hypocycloid. I have also obtained the equation of the envelope of the directrices (a curve of the fourth class) and the equation of the locus of the vertices (a curve of the eighth degree); but the results have not been published. In the solution of a problem in the Educational Times (proposed by himself), Cayley* proved that the locus of the foci of the parabolas which pass through three fixed points is a unicursal quintic passing through the two circular points at infinity, by showing that the co-ordinates of the focus of any such parabola may be explicitly expressed as rational functions of a parameter of the fifth degree. As the locus of the foci of a system of similar conics passing through three fixed points is not in general a unicursal curve, it is hardly likely that Cayley's method could be extended to the general case.

In this paper I give in the first place a general solution of the problem of the foci, using (exact) trilinear co-ordinates; and then I give a second solution for the special cases of an equilateral triangle, by employing a special triangle of reference.

2. Let the triangle of reference have for its vertices the three fixed points through which the conic is to pass. Let $(\alpha, \beta, \gamma)$ be the focus, $\mathrm{F} ;(p, q, r)$ be the directrix $;(x, y, z)$ be a point $\mathrm{P}$ on the conic; and let $M$ be the foot of the perpendicular from $P$ on the directrix. Take $a, b, c, \triangle$, to denote the lengths of the sides and the area of the triangle of reference, as usual, and let $e$ be the eccentricity of the conic. Then

$$
\begin{aligned}
& \mathrm{FP}^{2}=\frac{a b c}{4 \triangle^{2}}\left\{a \cos \mathrm{A}(x-a)^{2}+b \cos \mathrm{B}(y-\beta)^{2}+c \cos \mathrm{C}(z-\gamma)^{2}\right\} \\
& \mathrm{PM}= \pm \frac{p x+q y+r z}{\left(p^{2}+q^{2}+r^{2}-2 q r \cos \mathrm{A}-2 r p \cos \mathrm{B}-2, \mu q \cos \mathrm{C}\right)^{1 / 2}} ; \\
& \therefore \frac{a b c}{4 \triangle^{2}} \cdot\left\{a \cos \mathrm{A}(x-\alpha)^{2}+b \cos \mathrm{B}(y-\beta)^{2}+c \cos \mathrm{C}(z-\gamma)^{2}\right\} \\
& =e^{2} \cdot \frac{(p x+q y+r z)^{2}}{p^{2}+q^{2}+r^{2}-2 q r \cos \mathrm{A}-2 r p \cos \mathrm{B}-2 p q \cos \mathrm{C}}
\end{aligned}
$$

* Collected Mathematical Papers, vol. 7, p. 568. 
Now make this equation homogeneous in $x, y, z$, and find the conditions that the conic pass through the vertices of the triangle of reference. We have

$$
\begin{gathered}
a \cos \mathrm{A}(x-\alpha)^{2}+b \cos \mathrm{B}(y-\beta)^{2}+c \cos \mathrm{C}(z-\gamma)^{2} \\
=a \cos \mathrm{A} x^{2}+b \cos \mathrm{B} y^{2}+c \cos \mathrm{C} z^{2} \\
-(2 \alpha x \cdot a \cos \mathrm{A}+2 \beta y \cdot b \cos \mathrm{B}+2 \gamma z \cdot c \cos \mathrm{C}) \cdot \frac{a x+b y+c z}{2 \Delta} \\
+\left(\alpha^{2} a \cos \mathrm{A}+\beta^{2} b \cos \mathrm{B}+\gamma^{2} c \cos \mathrm{C}\right) \cdot \frac{(a x+b y+c z)^{2}}{4 \triangle^{2}} . \\
=\mathrm{P} x^{2}+\mathrm{Q} y^{2}+\mathrm{R} z^{2}+\ldots \text { (say). }
\end{gathered}
$$

Hence

$$
\begin{aligned}
\mathrm{P}= & a \cos \mathrm{A}-2 a a \cos \mathrm{A} \cdot \frac{a}{2 \triangle}+\left(a^{2} a \cos \mathrm{A}+\beta^{2} b \cos \mathrm{B}+\gamma^{2} c \cos \mathrm{C}\right) \cdot \frac{a^{2}}{4 \triangle^{2}} \\
=a \cos \mathrm{A} & \cdot \frac{(a a+b \beta+c \gamma)^{2}}{4 \triangle^{2}}-2 a \cdot a \cos \mathrm{A} \cdot \frac{a}{2 \triangle} \cdot \frac{a a+b \beta+c \gamma}{2 \triangle} \\
& +\left(a^{2} \cdot a \cos \mathrm{A}+\beta^{3} \cdot b \cos \mathrm{B}+\gamma^{2} \cdot \cos \mathrm{C}\right) \cdot \frac{a^{2}}{4 \triangle^{2}}
\end{aligned}
$$

and this expression may easily be reduced to

$$
\frac{a b c}{4 \triangle^{2}} \cdot\left(\beta^{2}+\gamma^{2}+2 \beta \gamma \cos \mathrm{A}\right)=\frac{a b c}{4 \triangle^{2}}, a_{1}^{2} \text { (say). }
$$

Let $\quad \mathrm{S}=p^{2}+q^{2}+r^{2}-2 q r \cos \mathrm{A}-2 r p \cos \mathrm{B}-2 p q \cos \mathrm{C}$, then the condition that the conic pass through the vertex $A$ is

$$
\frac{a b c}{4 \triangle^{2}} \cdot \frac{a b c}{4 \triangle^{2}} \cdot a_{1}{ }^{2}=\frac{e^{2} p^{2}}{\mathrm{~S}}
$$

Hence denoting $a b c / 4 \Delta^{2} e$ by $k$, we have

$$
\begin{aligned}
& p=k \sqrt{ } \mathbf{S} \cdot a_{1} \\
& q=k \sqrt{ } \mathbf{S} \cdot b_{1} \\
& r=k \sqrt{ } \mathbf{S} \cdot c_{1} .
\end{aligned}
$$

From these three equations we may eliminate the ratios $p: q: r$; for we have

$$
\begin{aligned}
& \quad k^{2} a_{1}{ }^{2}\left(p^{2}+q^{2}+r^{2}-2 q r \cos \mathrm{A}-2 r p \cos \mathrm{B}-2 p q \cos \mathrm{C}\right)=p^{2}, \\
& \therefore k^{2} a_{1}{ }^{2} \cdot k^{2} \mathrm{~S}\left(a_{1}{ }^{2}+b_{1}{ }^{2}+c_{1}{ }^{2}-2 b_{1} c_{1} \cos \mathrm{A}-2 c_{1} a_{1} \cos \mathrm{B}-2 a_{1} b_{1} \cos \mathrm{C}\right)=k^{2} \mathrm{~S} a_{1}{ }^{2} \\
& \therefore a_{1}{ }^{3}+b_{1}{ }^{2}+c_{1}{ }^{2}-2 b_{1} c_{1} \cos \mathrm{A}-2 c_{1} a_{1} \cos \mathrm{B}-2 a_{1} b_{1} \cos \mathrm{C}=1 / k^{2}=\left(4 \Delta^{2} e / a b c\right)^{2} \\
& 4 \mathrm{Vol.} 27
\end{aligned}
$$


Now if $p_{1}, p_{2}, p_{3}$ be the distances of a point from the vertices, it is easily seen geometrically that $a_{1}=p_{1} \sin A, b_{1}=p_{2} \sin \mathrm{B}, c_{1}=p_{9} \operatorname{sinC}$; hence the equation just obtained is a tri-focal equation of the locus of the foci.

Now let

$$
\begin{aligned}
& k_{1}=1 / k \\
& \lambda=a_{1}{ }^{2}+b_{1}{ }^{2}+c_{1}{ }^{2} \\
& \mu=b_{1}{ }^{2} c_{1} \cos ^{2} \mathrm{~A}+c_{1}{ }^{2} a_{1}{ }^{2} \cos ^{2} \mathrm{~B}+a_{1}{ }^{2} b_{1}{ }^{2} \cos ^{2} \mathrm{C} \\
& \nu=\sin \mathrm{B} \cos \mathrm{B} \sin \mathrm{C} \cos \mathrm{C} a_{1}{ }^{2}+\ldots \\
& \mathrm{I}=a \sin \mathrm{A}+\beta \sin \mathrm{B}+\gamma \sin \mathrm{C},
\end{aligned}
$$

then we have

$$
a_{1}{ }^{2}+b_{1}{ }^{2}+c_{1}{ }^{2}-2 b_{1} c_{1} \cos \mathrm{A}-2 c_{1} a_{1} \cos \mathrm{B}-2 a_{1} b_{1} \cos \mathrm{C}=k_{1}{ }^{2}
$$

$\therefore\left(\lambda-k_{1}^{2}\right)^{2}=4 \mu+8 a_{1} b_{1} c_{1}\left(a_{1} \cos \mathrm{B} \cos \mathrm{C}+b_{1} \cos \mathrm{C} \cos \mathrm{A}+c_{1} \cos \mathrm{A} \cos \mathrm{B}\right)$

$\therefore\left[\left(\lambda-k_{1}^{2}\right)^{2}-4 \mu\right]^{2}=64 a_{1}^{2} b_{1}^{2} c_{1}^{2}\left[a_{1}^{2} \cos ^{2} \mathrm{~B} \cos ^{2} \mathrm{C}+b_{1}{ }^{2} \cos ^{2} \mathrm{C} \cos ^{2} \mathrm{~A}+c_{1}^{2} \cos ^{2} \mathrm{~A} \cos ^{2} \mathrm{~B}\right.$

$$
\begin{aligned}
& \left.+2 \cos \mathrm{A} \cos \mathrm{B} \cos \mathrm{C}\left(b_{1} c_{1} \cos \mathrm{A}+c_{1} a_{1} \cos \mathrm{B}+a_{1} b_{1} \cos \mathrm{C}\right)\right] \\
& =64 a_{1}{ }^{2} b_{1}{ }^{2} c_{1}{ }^{2}\left[a_{1}{ }^{2} \cos ^{2} \mathrm{~B} \cos ^{2} \mathrm{C}+b_{1}{ }^{2} \cos ^{2} \mathrm{C}^{2} \cos ^{2} \mathrm{~A}+c_{1}{ }^{2} \cos ^{2} \mathrm{~A} \cos ^{2} \mathrm{~B}\right. \\
& \left.+\cos \mathrm{A} \cos \mathrm{B} \cos \mathrm{C}\left(\lambda-k_{1}{ }^{2}\right)\right]
\end{aligned}
$$

Since $\quad \cos ^{2} \mathrm{~B} \cos ^{2} \mathrm{C}+\cos \mathrm{A} \cos \mathrm{B} \cos \mathrm{C}=\sin \mathrm{B} \cos \mathrm{B} \sin \mathrm{C} \cos \mathrm{C}$, we have finally the equation

$$
\left[\left(\lambda-k_{1}^{2}\right)^{2}-4 \mu\right]^{2}=64 a_{1}^{2} b_{1}^{2} c_{1}^{2}\left(v-k_{1}^{2} \cos A \cos B \cos C\right) .
$$

If we substitute for $k_{1}$ its value $e I$, we have a homogeneous equation of the eighth degree in $a, \beta, \gamma$, but it may be shown that $\mathrm{I}^{2}$ is a factor.

\section{Reduction of the equation.}

We have

$$
\begin{aligned}
& \lambda^{2}=\left(a_{1}^{2}+b_{1}^{2}+c_{1}^{2}\right)^{2} \\
& =4\left(\alpha^{2}+\beta^{2}+\gamma^{2}+\beta \gamma \cos \mathrm{A}+\gamma \alpha \cos \mathrm{B}+\alpha \beta \cos \mathrm{C}\right)^{2} \\
& =4 \Sigma a^{4}+8 \Sigma a^{2} \beta^{2}+4 \Sigma \alpha^{2} \beta^{2} \cos ^{2} \mathrm{C}+8 \Sigma a^{2} \beta \gamma \cos B \cos C \\
& +8 \Sigma \alpha^{3} \beta \cos C+8 \Sigma \alpha^{3} \beta \gamma \cos \mathrm{A} \\
& \mu=\Sigma\left(\gamma^{2}+\alpha^{2}+2 \gamma \alpha \cos B\right)\left(\alpha^{2}+\beta^{2}+2 \alpha \beta \cos C\right) \cos ^{2} A \\
& =\Sigma \alpha^{4} \cos ^{2} \mathrm{~A}+\Sigma \alpha^{2} \beta^{2} \cdot \Sigma \cos ^{2} \mathrm{~A}+2 \Sigma \alpha^{3} \beta \cos C \cos ^{2} \mathrm{~A} \\
& +2 \Sigma^{2} \beta \gamma \cos A \sin ^{2} A \\
& \therefore \quad \lambda^{2}-4 \mu=4 \Sigma a^{4} \sin ^{2} A+4 \Sigma \alpha^{2} \beta^{2}\left(\sin ^{2} A+\sin ^{2} B\right) \\
& +8 \Sigma \alpha^{3} \beta \cos C \sin ^{2} A+8 \Sigma \alpha^{2} \beta \gamma\left(\sin B \sin C-\cos A \sin ^{2} A\right) \text {. }
\end{aligned}
$$


Again

$$
\begin{aligned}
\mathrm{I}^{2}\left(\alpha^{2}+\beta^{2}+\gamma^{2}\right)=\Sigma a^{4} \sin ^{2} A+\Sigma \alpha^{2} \beta^{2}\left(\sin ^{2} A\right. & \left.+\sin ^{2} \mathrm{~B}\right) \\
& +2 \Sigma \alpha^{3} \beta \sin A \sin B+2 \Sigma \alpha^{2} \beta \gamma \sin B \sin \mathrm{C} \\
\therefore \quad \lambda^{2}-4 \mu & =4 \mathrm{I}^{2}\left(\alpha^{2}+\beta^{2}+\gamma^{2}\right)-8\left[\Sigma \alpha^{3} \beta \sin A \sin C \cos \mathrm{A}\right. \\
& \left.+\Sigma \alpha^{2} \beta \gamma \cos A \sin ^{2} \mathrm{~A}\right]
\end{aligned}
$$

Now let

then

$$
\begin{aligned}
& P=\sin A \cos A \alpha^{2}+\sin B \cos B \beta^{2}+\sin C \cos C \gamma^{2} \\
& Q=\sin A \beta \gamma+\sin B \gamma \alpha+\sin C a \beta,
\end{aligned}
$$

$$
\lambda^{2}-4 \mu=4 \mathrm{I}^{2}\left(\alpha^{2}+\beta^{2}+\gamma^{2}\right)-8 \mathrm{PQ} ;
$$

and we may easily show that

$$
\begin{aligned}
v & =\Sigma \sin \mathrm{B} \cos \mathrm{B} \sin \mathrm{C} \cos \mathrm{Ca}_{1}{ }^{2}, \\
& =\sin \mathrm{A} \sin \mathrm{B} \sin \mathrm{C} \cdot \mathrm{P}+\cos \mathrm{A} \cos \mathrm{B} \cos \mathrm{CI}^{2}
\end{aligned}
$$

Again

$$
\begin{aligned}
a_{1}^{2} b_{1}{ }^{2} c_{1}{ }^{2}=\Sigma a^{4} \beta^{2}+\alpha^{2} \beta^{2} \gamma^{2}(2+8 \cos A \cos B \cos C)+2 \Sigma \alpha^{4} \beta \gamma \cos A \\
+2 \Sigma \alpha^{3} \beta^{2} \gamma(\cos B+2 \cos A \cos C)+2 \Sigma \beta^{3} \gamma^{3} \cos A ;
\end{aligned}
$$

and we may show that

$$
\sin A \sin B \sin C \cdot a_{1}{ }^{2} b_{1}{ }^{2} c_{1}{ }^{2}=P Q^{2}+I^{2} . \Sigma \beta^{2} \gamma^{2} \sin A \cos A
$$

$$
\text { If } Q^{\prime}=\cos A \cdot \beta \gamma+\cos B \cdot \gamma \alpha+\cos C \cdot \alpha \beta \text {, then }
$$

$$
\Sigma \beta^{2} \gamma^{2} \sin A \cos A=Q Q^{\prime}-\alpha \beta \gamma I ;
$$

hence if $p=\cot \mathrm{AcotB} \cot \mathrm{C}$

$$
\text { and } R=a^{2}+\beta^{2}+\gamma^{2} \text {, so that } \lambda=2\left(R+Q^{\prime}\right) \text {, }
$$

the equation, after $I^{2}$ is divided out, may be written in the form

$$
\begin{aligned}
\mathrm{I}^{2}\left[4 \mathrm{R}-2 \lambda e^{2}+e^{4} \mathrm{I}^{2}\right]^{2}-16 \mathrm{PQ}\left[4 \mathrm{R}-2 \lambda e^{2}+e^{4} \mathrm{I}^{2}\right] \\
=64 \mathrm{P}\left[\mathrm{QQ} \mathrm{Q}^{\prime}-a \beta \gamma \mathrm{I}\right]+64 p\left(1-\sigma^{2}\right)\left[\mathrm{PQ}^{2}+\mathrm{I}^{3}\left(\mathrm{QQ}^{\prime}-\alpha \beta \gamma \mathrm{I}\right)\right],
\end{aligned}
$$

or, since $\quad 64 \mathrm{PQQ}^{\prime}+16 \mathrm{PQ}\left(4 \mathrm{R}-2 \lambda e^{2}\right)=64\left(1-e^{2}\right) \mathrm{PQ}\left(\mathrm{R}+\mathrm{Q}^{\prime}\right)$

in the form

$$
\begin{aligned}
\mathrm{I}^{2}\left[4 \mathrm{R}-2 \lambda \sigma^{2}+e^{4} \mathrm{I}^{2}\right]^{2}-16 e^{4} \mathrm{PQI} I^{2}+64 \alpha \beta \gamma \mathrm{PI} \\
=64\left(1-e^{2}\right)\left[\mathrm{PQ}\left(\mathrm{R}+\mathrm{Q}^{\prime}\right)+p\left(\mathrm{PQ}^{2}+\mathrm{QQ}^{\prime} \mathrm{I}^{2}-\alpha \beta \gamma \mathrm{I}^{2}\right)\right] .
\end{aligned}
$$

This is the equation of the locus of the foci, and represents a bi-circular sextic. For $P=0, Q=0$ and $R+Q^{\prime}=0$ all represent circles, hence the straight line at infinity meets the sextic only in the circular points. But these points are double and not triple points on the locus, for it may be shown without difficulty that the curve touches the straight line at infinity at the circular points. 
If $e=1$, the case of the parabola, $I$ is again a factor, and the locus reduces to a curve of the fifth degree, passing once through each of the circular points at infinity. In this case the straight line at infinity meets the curve in the circular points and in its intersections with $a, \beta$ and $\gamma$; hence, in the case of the parabola, there are three real asymptotes, which are parallel to the sides of the triangle of reference. In the general case there can obviously be no real asymptotes.

\section{Double Points.}

Taking the equation in the irrational form

$$
a_{1}^{2}+b_{1}{ }^{2}+c_{1}{ }^{2}-2 b_{1} c_{1} \cos \mathrm{A}-2 c_{1} a_{1} \cos \mathrm{B}-2 a_{1} b_{1} \cos \mathrm{C}=e^{2} \mathrm{~T}^{2},
$$

we may show that

$$
\begin{aligned}
& \sin (\mathrm{A}-\mathrm{B})\left[a_{1}{ }^{2}+b_{1}{ }^{2}+c_{1}{ }^{2}-2 b_{1} c_{1} \cos \mathrm{A}-2 c_{1} a_{1} \cos \mathrm{B}-2 a_{1} b_{1} \cos \mathrm{C}-e^{2} \mathrm{~T}^{2}\right] \\
& =\left(b_{1}{ }^{2}-a_{1}{ }^{2}\right) \sin \mathrm{C}+\left(c_{1}{ }^{2}-e^{2} \mathrm{I}^{2}\right) \sin (\mathrm{A}-\mathrm{B}) \\
& +2\left(a_{1} \sin \mathrm{A}-b_{1} \sin \mathrm{B}-c_{1}\right)\left(a_{1} \cos \mathrm{B}+b_{1} \cos \mathrm{A}\right) \text {; } \\
& \text { hence, putting } \mathrm{L}=a_{1}{ }^{2} \cos ^{2} \mathrm{~B}-b_{1}{ }^{2} \cos ^{2} \mathrm{~A} \\
& \mathbf{M}=\left(b_{1}{ }^{2}-a_{1}{ }^{2}\right) \sin \mathrm{C}+\left(c_{1}{ }^{2}-e^{2} \mathbf{I}^{2}\right) \sin (\mathbf{A}-\mathrm{B}) \text {, }
\end{aligned}
$$

it is not difficult to show that the rationalized form of the equation may be written in the form

$$
\mathrm{UL}^{2}+\mathrm{VLM}+\mathrm{WM}^{2}=0 .
$$

where $\mathrm{U}, \mathrm{V}, \mathrm{W}$, are rational integral functions of $\alpha, \beta, \gamma$. It follows from this that the intersections of $L$ and $M$ (other than the circular points at infinity, for $\mathrm{I}^{2}$ is a factor of the rational equation obtained in this way) are double points on the sextic. We thus obtain a pair of double points, and by symmetry two other pairs, so that, with the two circular points at infinity, the curve has eight double points. It may be shown that in general there are no more double points, hence the deficiency of the sextic is in general 2. In the case of the parabola the circular points at infinity cease to be double points, but the other six double points are retained, so that the deficiency of the quintic is zero, that is, it is unicursal.

The curve $L=0$ is easily seen to be a circle ; in fact, it is obvious geometrically that it is the locus of a point whose distances from the vertices $A$ and $B$ are proportional to the segments made on the side $\mathrm{AB}$ by the perpendicular from the opposite vertex $\mathrm{C}$.

It should be noted that this and the other two corresponding circles are independent of $e$, and hence they form the locus of the double points of the sextic for varying values of the eccentricity. 
It may be shown that $\mathrm{M}$ resolves into factors, namely, the straight line at infinity, $I$, and the straight line

$$
2(\alpha \cos \mathrm{B}-\beta \cos \mathrm{A})-e^{2} \mathrm{I} \sin (\mathrm{A}-\mathrm{B})=0 .
$$

This latter straight line is parallel to the line joining the vortex $\mathrm{C}$ to the circum-centre.

When $A=B$ the circle $L$ degenerates into the straight line at infinity and the perpendicular from $\mathrm{C}$ on the side $\mathrm{AB}$; so that in this case $L$ and $M$ become identical. The intersections of $L$ and $M$, however, remain definite, and may be obtained by putting $\mathrm{B}=\mathrm{A}+h$, and taking the limit when $h$ is zero.

In this way $I$ find that, for the case $A=B$, the two double points in question are given by

$$
a=\beta \text { and } e^{2}(\gamma+2 a)^{2}=4\left(\gamma^{2}+\gamma a+2 a^{2}\right) \text {. }
$$

In this case the double points are real or imaginary, according as $e^{2}>$ or $<7 / 4$.

\section{Special Case of the Equilateral Triangle.}

\section{The Triangle of Reference.}

For any triangle of reference the equation of a circumscribed conic is

$$
\lambda \beta \gamma+\mu \gamma \alpha+\nu \alpha \beta=0,
$$

and we may show that the condition that this system of conics be similar is*

$$
\Sigma\left(\lambda^{2} \sin ^{2} \mathrm{~A}-2 \mu \nu \sin \mathrm{B} \sin \mathrm{C}\right)=t^{2}(\lambda \cos \mathrm{A}+\mu \cos \mathrm{B}+\nu \cos \mathrm{C})^{2}
$$

where $t$ is the tangent of the angle between the asymptotes; or

$$
\Sigma\left(\lambda^{2}-2 \mu \nu \cos \mathrm{A}\right)=\boldsymbol{s}^{2}(\lambda \cos \mathrm{A}+\mu \cos \mathrm{B}+\nu \cos \mathrm{C})^{2}
$$

where $s$ is the secant of the angle between the asymptotes.

Now, suppose the triangle of reference to be equilateral and transform to a new triangle of reference, which has for its vertices the centre of the triangle and the circular points at infinity, by putting

$$
\begin{aligned}
\alpha & =\omega x+\omega^{2} y+z, \\
\beta & =\omega^{2} x+\omega y+z, \\
\gamma & =x+y+z,
\end{aligned}
$$

where $\omega$ is a cube root of unity.

* See the paper entitled, On some curves, etc., referred to above. 


\section{4}

The equation of the conic now becomes

where

$$
l\left(x^{2}-y z\right)+m\left(y^{2}-z x\right)+n\left(z^{2}-x y\right)=0 .
$$

$$
\begin{aligned}
l & =\omega^{2} \lambda+\omega \mu+\nu, \\
m & =\omega \lambda+\omega^{2} \mu+v, \\
n & =\lambda+\mu+v ;
\end{aligned}
$$

and the condition for similarity becomes

$$
4 \ln =s^{2} n^{2} \text {. }
$$

It may be noted in passing that the condition for similarity is the tangential equation of a circle concentric with the circum-circle; hence if the quadratic transformation $a=1 / a$, etc., be applied to system of similar circumscribed conics, the resulting system of straight lines will all touch this circle. This theorem is given by Schoute (loc. cit.).

If the original triangle of reference be not taken to be equilateral, and a transformation be made to a new triangle of reference, having for its vertices the ortho-centre of the triangle and the circular points at infinity, the condition for similarity may be reduced to the same simple form ( $\left.4 l m=R^{2} n^{2}\right)$; but the equation of the circumscribed conic is not so simple, and the eliminations involved in finding the locus of the foci seem too complicated to be manageable.

Without loss of generality we may put $n=1$, and take the equation of the conic in the form

$$
\mathrm{U} \equiv l\left(x^{2}-y z\right)+m\left(y^{2}-z x\right)+z^{3}-x y=0
$$

and the condition for similarity in the form $4 l m=8^{2}$.

The equation of an axis is *

$$
2(2 l x-y-m z)=2 l(-x+2 m y-l z) ;
$$

and that of the tangents from the vertex, $y=0, z=0$ (one of the circular points at infinity), is

$$
\begin{gathered}
\mathrm{U}_{1}^{\prime}{ }^{3}=4 \mathrm{UU}_{1}, \text { which reduces to } \\
\left(1-z^{2}\right) y^{2}+\left(m^{2}-4 l\right) z^{2}+\left(4 l^{2}+2 m\right) y z=0 .
\end{gathered}
$$

Putting $s=2 k$, we have, to determine the foci,

$$
\begin{gathered}
\left(1-4 k^{2}\right) y^{2}+\left(m^{2}-4 l\right) z^{2}+\left(4 l^{2}+2 m\right) y z=0, \\
l(1+2 k) x-k(1+2 k) y+\left(l^{2}-k m\right) z=0 .
\end{gathered}
$$

- Bee the paper entitlod, On the envelope of the ases, etc, referrod to above. 
On interchanging $l$ and $m$, and $x$ and $y$, we get the same axis, and the tangents from the other vertex (circular point at infinity), on which the same foci must lie; the other axis is got by changing the sign of 8 .

The above equations may be written

$$
\begin{gathered}
4 y z \cdot l^{2}+z^{2} \cdot m^{2}-4 z^{2} \cdot l+2 y z \cdot m+\left(1-4 k^{2}\right) y^{2}=0, \\
\quad \approx \cdot l^{2}+(1+2 k) x \cdot l-k z \cdot m-k(1+2 k) y=0
\end{gathered}
$$

or, putting $m=k^{2} / l$,

$$
\begin{array}{r}
4 y z \cdot l^{4}-4 z^{2} \cdot l^{3}+\left(1-4 k^{2}\right) y^{2} \cdot l^{2}+2 y z k^{2} \cdot l+k^{4} z^{2}=0 \\
z \cdot l^{3}+(1+2 k) x \cdot l^{2}-k(1+2 k) y \cdot l-k^{3} z=0 .
\end{array}
$$

Hence we have

$4 y z \cdot l^{3}+(k-4) z^{2} \cdot l^{2}+\left[\left(1-4 k^{2}\right) y^{2}+k(1+2 k) x z\right] \cdot l+k^{2}(1-2 k) y z=0$, and, by eliminating the first and last terms between the last two equations

$\left[(k-4) z^{2}-4(1+2 k) x y\right] l^{3}+\left[(1+2 k)^{2} y^{2}+k(1+2 k) x z\right] \cdot l+k^{2}(1+2 k) y z=0$, $(1+2 k) y z \cdot l^{2}+\left[k(k-4) z^{2}+\left(1-4 k^{2}\right) x y\right] \cdot l+k^{2}(1+2 k) x z=0$.

Interchanging $x$ and $y$, and $l$ and $m$, and putting $m=k^{2} / l$ in the second last equation we get

$(1+2 k) x z . l^{2}+\left[(1+2 k)^{2} x^{2}+k(1+2 k) y z\right] . l+k^{2}\left[(k-4) z^{2}-4(1+2 k) x y\right]=0 ;$ and, finally, eliminating $l, l, k^{2}$, dialytically between the last three equations, we have

$$
\begin{array}{ccc|}
(1+2 k) y z & k(k-4) z^{2}+\left(1-4 k^{2}\right) x y & (1+2 k) x z \\
(k-4) z^{2}-4(1+2 k) x y & (1+2 k)\left[(1+2 k) y^{2}+k x z\right] & (1+2 k) y z \\
(1+2 k) x z & (1+2 k)\left[(1+2 k) x^{2}+k y z\right] & (k-4) z^{2}-4(1+2 k) x y
\end{array}
$$

which is the equation of the locus.

\section{Reduction of the equation.}

In the case of the original (equilateral) triangle the conic degenerates into a side and another straight line if $\lambda=0$ or $\mu=0$ or $\nu=0$, and in each of these cases the focus lies on a side. It is easy to show that the six points on the sides of the triangle of reference, obtained in this way, lie on the circle

$$
\left(4 k^{2}-1\right)(x y+y z+z x)-\left(k^{2}-1\right)(x+y+z)^{2}=0 ;
$$

and this circle, transformed to the new triangle of reference, becomes

$$
\left(k^{2}+2\right) z^{2}-\left(4 k^{2}-1\right) x y=0 .
$$

This result is useful in the reduction of the equation. 
Now if the determinant obtained at the end of the last paragraph be multiplied out, and the terms containing $x^{3}$ and $y^{3}$ be collected, we find that these terms are

$$
-4(2 k+1)^{3}\left(x^{3}+y^{3}\right) z\left[z^{2}+(2 k+1) x y\right]
$$

and if, from the expanded form of the determinant, we subtract

$$
-4(2 k+1)^{3} z\left[z^{3}+(2 k+1) x y\right]\left[x^{3}+y^{3}+z^{3}-3 x y z\right],
$$

the remainder is

$$
\begin{aligned}
& -\left(k^{2}+2\right)\left(k^{2}-44 k-2\right) z^{6} \\
& +(2 k+1)\left(48 k^{3}-102 k^{2}+132 k-24\right) x y z^{4} \\
& +16\left(4 k^{2}-1\right)(2 k+1)^{2} x^{3} y^{3} \\
& -(2 k+1)^{2}\left(108 k^{2}-72 k+45\right) x^{2} y^{2} z^{2},
\end{aligned}
$$

This last expression resolves into factors, namely, it is equal to

$\left[\left(4 k^{2}-1\right) x y-\left(k^{2}+2\right) z^{2}\right] \times$

$\left[16(2 k+1)^{2} x^{2} y^{2}-(2 k+1)(46 k-13) x y z^{2}+\left(k^{2}-44 k-2\right) z^{4}\right]:$

Again, putting

$$
\begin{aligned}
\lambda & =\left(4 k^{2}-1\right) x y-\left(k^{2}+2\right) z^{2} \\
\mu & =(2 k+1) x y+z^{2},
\end{aligned}
$$

we may show that

$$
\begin{aligned}
& 16(2 k+1)^{2} x^{2} y^{2}-(2 k+1)(46 k-13) x y z^{2}+\left(k^{2}-44 k-2\right) z^{4} \\
& =\frac{1}{(k+1)^{2}} \cdot\left[\lambda^{2}+21(2 k+1) \lambda \mu-36(2 k+1)(k-1) \mu^{2}\right]
\end{aligned}
$$

Hence, if

$$
\mathrm{P}=x^{3}+y^{3}+z^{3}-3 x y z,
$$

the equation of the locus is

$$
\begin{gathered}
\frac{1}{(k+1)^{2}} \lambda \cdot\left[\lambda^{2}+21(2 k+1) \lambda \mu-36(2 k+1)(k-1) \mu^{2}\right. \\
-4(1+2 k)^{3} z \mu \mathrm{P}=0 .
\end{gathered}
$$

\section{Special cases.}

The relation between $k$ and $e$ is $2 k=e^{2} /\left(2-e^{2}\right)$; hence for the parabola $2 k=1$; and thus in this case $\lambda$ reduces to $-\left(k^{2}+2\right) z^{2}$, and $z$ is a factor in the equation.

In the case of the equilateral hyperbola $k=\infty$, and the equation becomes

$$
64 x y z \mathrm{P}-\left(4 x y-z^{2}\right)\left[\left(4 x y-z^{2}\right)^{2}+84 x y\left(4 x y-z^{2}\right)-288 x^{2} y^{2}\right]=0,
$$

which may also be written

$$
64 x y z\left[x^{3}+y^{3}-\frac{1}{8} z^{3}+\frac{3}{2} x y z\right]+\left(z^{2}-4 x y\right)^{\prime \prime}\left(z^{2}-16 x y\right)=0 .
$$




\section{Multiple Points.}

The equation of the curve, multiplied out, is

$$
\mathrm{K} \equiv \mathrm{A} z^{6}+\mathrm{B} z^{4} x y+\mathrm{C} z^{3}\left(x^{3}+y^{3}\right)+\mathrm{D} z^{2} x^{2} y^{2}+\mathrm{E} z x y\left(x^{3}+y^{3}\right)+\mathrm{F} x^{3} y^{3}=0,
$$

where

$$
\begin{aligned}
& \mathrm{A}=-(k-4)^{3} \\
& \mathrm{~B}=(k-4)(2 k+1)\left(16 k^{2}-38 k+4\right) \\
& \mathrm{C}=-4(2 k+1)^{3} \\
& \mathrm{D}=-3(2 k+1)^{2}\left(20 k^{2}-40 k+11\right) \\
& \mathrm{E}=-4(2 k+1)^{4} \\
& \mathrm{~F}=16(2 k+1)^{3}(2 k-1) .
\end{aligned}
$$

Hence the double points are given by

$$
\begin{aligned}
& \frac{\partial \mathrm{K}}{\partial x} \equiv \mathrm{B} z^{4} y+3 \mathrm{C} z^{3} x^{2}+2 \mathrm{D} z^{2} x y^{2}+4 \mathrm{E} z x^{3} y+\mathrm{E} z y^{4}+3 \mathrm{~F} x^{2} y^{3}=0 \\
& \frac{\partial \mathrm{K}}{\partial y} \equiv \mathrm{B} z^{4} x+3 \mathrm{C} z^{3} y^{2}+2 \mathrm{D} z^{2} x^{2} y+4 \mathrm{E} z x y^{3}+\mathrm{E} z x^{4}+3 \mathrm{~F} x^{3} y^{2}=0 .
\end{aligned}
$$

Multiplying by $x$ and $y$ and subtracting, we get

$$
\begin{aligned}
& 3 z\left(x^{3}-y^{3}\right)\left(\mathrm{C} z^{2}+\mathrm{E} x y\right)=0 \\
& \mathrm{C} z^{2}+\mathrm{E} x y=0 \text { reduces to } \\
& z^{2}+(2 k+1) x y=0, \text { which gives no double points except at }
\end{aligned}
$$
the vertices $(x=0, z=0)$ and $(y=0, z=0)$.

If we put $y=x$ in $\partial \mathrm{K} / \partial x$ we get

$$
x\left(\mathrm{~B} z^{4}+3 \mathrm{C} z^{3} x+2 \mathrm{D} z^{2} x^{2}+5 \mathrm{E} z x^{3}+3 \mathrm{~F} x^{4}\right)
$$

and this expression contains

us a factor.

$$
(k-4) z^{2}-(2 k+1) z x-4(2 k+1) x^{2}
$$

As the square of this last expression is a factor in $\mathrm{K}$, when we put $y=x$, it follows that we thus obtain two double points; and two other pairs of double points are got by putting $y=\omega x$ and $y=\omega^{2} x$. Hence there are eight double points in all.

It may be noted that if we transform the equation

$$
(k-4) z^{2}-(2 k+1) z x-4(2 k+1) x^{2}=0
$$

to the original (equilateral) triangle of reference, and put $2 k=e^{2} /\left(2-e^{2}\right)$, it becomes

$$
e^{2}(\gamma+2 \alpha)^{2}=4\left(\gamma^{2}+\gamma \alpha+2 \alpha^{2}\right)
$$

and thus agrees with the result obtained for the double points in the limiting case when $B=A$ (\$). 


\section{Verification.}

A verification of certain of the above formulae, in the case of the equilateral triangle, may be obtained by consideration of an ellipse circumscribed about an equilateral triangle in such a way that the principal axis coincides with the perpendicular from a vertex of the triangle on the opposite side. It may be shown that the co-ordinates of the real foci of the ellipse are proportional to $\left[4-3 e^{2}, 4-3 e^{2}, 2\left(3 e^{2}+6 e+2\right)\right]$ and $\left[4-3 e^{2}, 4-3 e^{2}, 2\left(3 e^{2}-6 e+2\right)\right]$; and when the first of these points is transformed to the new triangle of reference, it becomes $[e(3 e+4), e(3 e+4), 4(e+1)]$. Hence if $p=z / x=4\left(e+1 /\left(3 e^{2}+4 e\right)\right.$, we have the equation

$$
\left[\left(3 e^{2}+4 e\right) p-4(e+1)\right]\left[\left(3 e^{2}-4 e\right) p-4(-e+1)\right]=0,
$$

or, putting

$$
2 k=e^{2} /\left(2-e^{2}\right),
$$

$$
k(k-4) p^{2}+2 k(2 k+1) p-\left(4 k^{2}-1\right)=0 .
$$

Now if, in the expanded form of the sextic given above, we put $y=x, z / x=p$, we find that the expression

$$
k(k-4) p^{2}+2 k(2 k+1) p-\left(4 k^{2}-1\right)
$$

is a factor, the other factor being a complete square, namely the square of

$$
(k-4) p^{2}-(2 k+1) p-4(2 k+1) \text {. }
$$

For the purpose of tracing the curves for special values of $k$, it is convenient to refer it to rectangular axes. This is most simply done by taking for origin the centre of the original (equilateral) triangle of reference, and for axes, lines parallel and perpendicular to one of the sides. The equations for transforming to these axes from the triangle of reference, which has for its vertices the centre of the original triangle of reference and the circular points at infinity, are

$$
\left.\begin{array}{l}
x=-\frac{1}{2 \sqrt{3}}\left(\omega^{2}-\omega\right) u+\frac{1}{3} v \\
y=-\frac{1}{2 \sqrt{3}}\left(\omega-\omega^{2}\right) u+\frac{1}{2} v \\
z=1
\end{array}\right\}
$$$$
\text { whence } \quad x+y=v
$$$$
x y=\frac{1}{2}\left(u^{2}+v^{2}\right)
$$$$
\omega x+\omega^{2} y=-\frac{\sqrt{ } 3}{2} u-\frac{1}{2} v
$$$$
\omega^{2} x+\omega y=+\frac{\sqrt{3}}{2} u-\frac{1}{2} v
$$ 
I give rough tracings of the locus in four special cuses, viz., $2 k=1$, the parabola (Fig. 1); $k=\infty$, the equilateral hyperbola

Fig. I

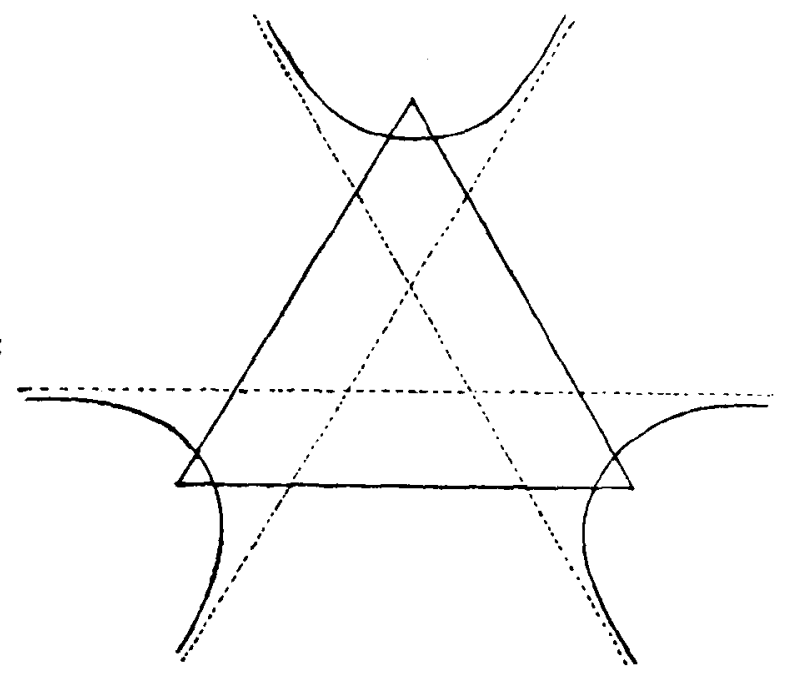

(Fig. 2); $k=4$, when three double points unite to give a triple point (Fig. 3); and $k=-1$, when the locus breaks up into the

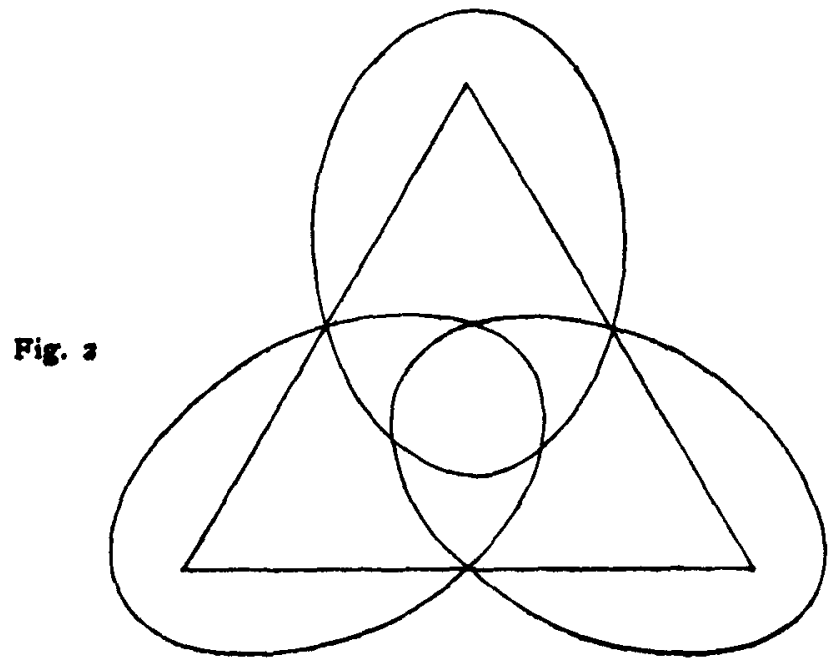


circum-circle and a unicursal quartic (Fig. 4). The circum-circle in the last case is the locus of the focus of a series of hyperbolas, of

Fig. 3

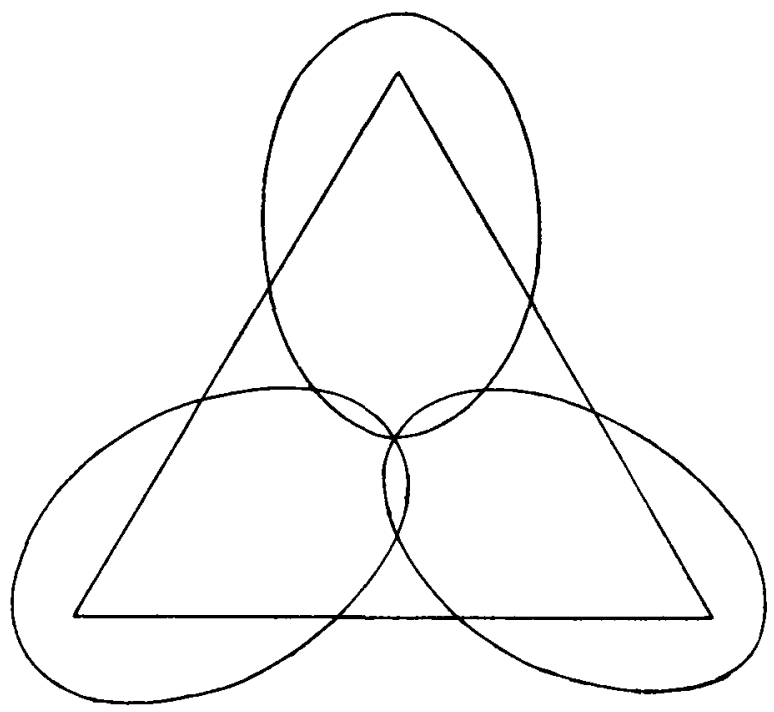

eccentricity 2 , the corresponding directrices of which pass through the centre of the triangle.

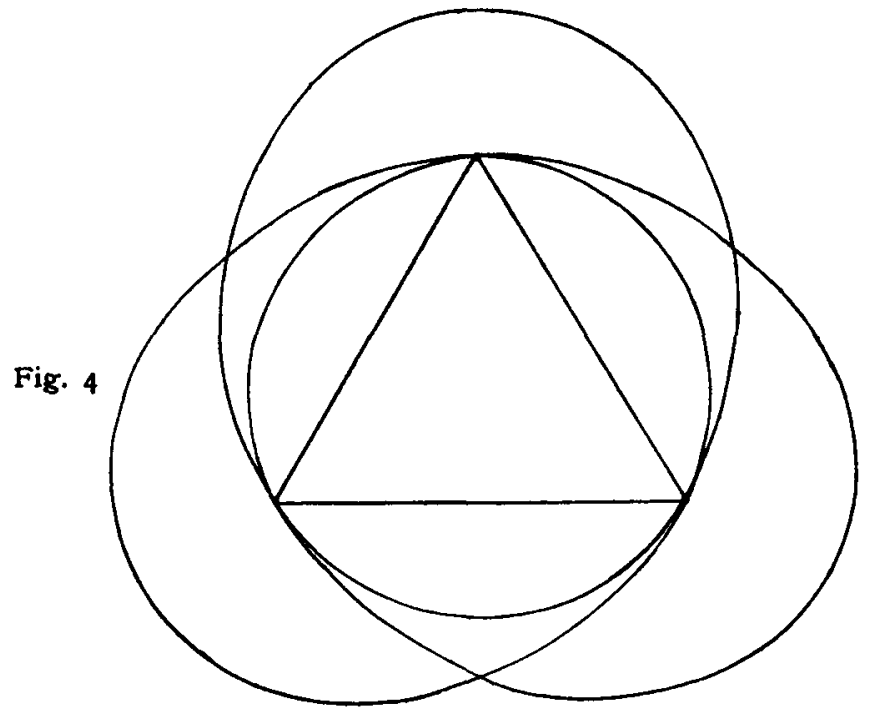

